\title{
Lossless Compression of Medical Image Sequences Using a Resolution Independent Predictor and Block Adaptive Encoding
}

Original Scientific Paper

\author{
Urvashi Sharma \\ Department of Electronics and Communication Engineering \\ Jaypee University of Information Technology \\ survashi2793@gmail.com

\section{Meenakshi Sood} \\ Department of Electronics and Communication Engineering \\ Jaypee University of Information Technology \\ meenakshi.sood@juit.ac.in

\section{Emjee Puthooran} \\ Department of Electronics and Communication Engineering \\ Jaypee University of Information Technology \\ emjee.puthooram@juit.ac.in
}

\begin{abstract}
The proposed block-based lossless coding technique presented in this paper targets at compression of volumetric medical images of 8-bit and 16-bit depth. The novelty of the proposed technique lies in its ability of threshold selection for prediction and optimal block size for encoding. A resolution independent gradient edge detector is used along with the block adaptive arithmetic encoding algorithm with extensive experimental tests to find a universal threshold value and optimal block size independent of image resolution and modality. Performance of the proposed technique is demonstrated and compared with benchmark lossless compression algorithms. BPP values obtained from the proposed algorithm show that it is capable of effective reduction of inter-pixel and coding redundancy. In terms of coding efficiency, the proposed technique for volumetric medical images outperforms CALIC and JPEG-LS by $0.70 \%$ and $4.62 \%$, respectively.
\end{abstract}

Keywords - adaptive encoding, lossless compression, predictive coding, resolution independent gradient edge detector, volumetric data compression

\section{INTRODUCTION}

With the advancement of digital medical imaging and scanning technologies, a huge amount of digital image data including MRI, CT, X-RAY, etc., is produced by various medical associations and hospitals. Images produced by advanced scanning techniques are of high quality with large image resolution and high bit depth of up to 16 bits. Such data is a series of image sequences and these medical image sequences necessitate large storage space [1], [2]. Medical applications like telemedicine demand effective storage and a high transmission speed of images between healthcare associations [3]. To preserve storage space and bandwidth, an efficient image compression technique is necessary. Compression reduces the image size by removing spatial and coding redundancy from the image. Different types of medical images with varying resolution, modality and bit depth require a common compression technique. Lossy com- pression techniques provide high compression at the expense of image quality due to loss of information [4]. Lossless compression is mostly preferred for medical images, especially for diagnostic purposes, since there is no loss of information and the recovered image is exactly the same as the original image [5]. Transform based encoding techniques, integer wavelet transforms (IWT), fractal compression, and linear predictive coding [5-9] are different lossless compression techniques available in literature. The 2D lossless image compression techniques JPEG [10] and JPEG2000 [11], [12] are based on DCT and DWT transform techniques. However, the performance of transform based lossless compression technique is not appreciable for medical images. The predictive coding techniques perform well among lossless coding techniques for medical images as they provide efficient compression in terms of bits per pixel (BPP). CALIC [13] and JPEG-LS [14] are predictive coding standards for lossless compression of images. JPEG-LS is 
a low complexity lossless compression algorithm, which consists of two independent stages called prediction and encoding. It is based on the LOCO algorithm and makes use of the standard median edge predictor (MED) predictor. The MED predictor removes spatial redundancy from the image and it can be easily implemented but its coding efficiency is not very high. After prediction, a Golomb code is used for entropy encoding of a residual image. A simple MED predictor and a Golomb encoder make the implementation of JPEG-LS less complex. CALIC is another predictive coding lossless standard that uses the gradient adaptive predictor (GAP) for prediction and arithmetic encoding for residual image encoding. GAP efficiency in terms of BPP is high but it is computationally more complex than the MED predictor.

Many researchers have worked on predictive coding techniques for lossless compression of medical images. Avramovic and Savic proposed a predictive algorithm for the estimation of local gradients and detection of edges. Entropy analysis for different predictors is done after prediction for different images like $C T$ and MRI [15]. Owen Zhao et al. proposed an efficient lossless image compression scheme called super-spatial structure prediction [16]. The efficiency of super-spatial prediction is high for image regions with considerable structure components. Al-Khafaji and Al-Mahmood proposed a simple fast lossless image compression method based on integrates multi-resolution coding along with polynomial approximation of linear based to decompose image signals followed by efficient coding for compressing medical images [17]. Jovanovic and Lorentz proposed a new lossless predictive coding method in which a predictor based on previously known pixel values is used to determine the order in which pixels are visited. Tests were performed on benchmark images and the results give similar or better results than standard algorithms like JPG2000 and median compression [18]. Song et al. proposed adaptive block size based spatial prediction to predict blocks and before quantization, a residual image is transformed by the lossless Hadamard transform to improve the recovered image quality [19]. Song et al. proposed an efficient algorithm based on irregular segmentation and region-based prediction to improve compression rates for lossless compression of medical images. $A$ hybrid method of geometry-adaptive partitioning and quad-tree partitioning is proposed to achieve adaptive irregular segmentation for medical images [20].

This paper presents an efficient coding solution based on predictive coding technique for lossless compression of medical images with varying image resolutions, modalities and bit depth. The proposed approach of predictive coding includes the resolution independent gradient edge detector (RIGED) and block adaptive arithmetic encoding (BAAE) for efficient compression independent of medical image resolutions and modalities. The existing gradient edge detection (GED) [21] has a demerit of threshold selection for prediction removed by RIGED providing an optimum threshold value for spatial redun- dancy removal. For removing spatial redundancy efficiently, an optimum value of the threshold should be selected to minimize the entropy value of the residual after prediction. No specific method is reported in literature for threshold value selection and the existing predictors are also dependent on resolution and modality. An optimal threshold value that provides minimum entropy for varying image modalities and resolutions is provided by the proposed RIGED. For the purpose of removing coding redundancy, BAAE is designed that provides optimal block size for blocking the residual before applying an arithmetic encoder. The proposed technique is validated for 8-bit depth images along with 16-bit depth images.

The rest of the paper is structured as follows: Section 2 describes the dataset used for this paper and a general overview of a predictive coding technique along with the details of the proposed block adaptive arithmetic coding (BAAE) and RIGED methodology. Lossless compression results on medical images are presented in Section 3, whereas Section 4 concludes the paper.

\section{MATERIALS AND METHODS}

\subsection{DATASET}

The proposed algorithm is tested on a set of MRI and CT medical sequence images. All images in test set I are of 8-bit depth. 16-bit depth images are contained in test set II. CT and MRI medical images in test set I with the resolutions of $256 \times 256$ and $512 \times 512$ are collected from three different sources presented in Table 1. CT and MRI medical images with the $256 \times 256$ resolution are collected from the Center for Image Processing Research (CIPR) [22]. MR images with the $512 \times 512$ resolution are obtained from Toronto Tri-Hospital MR Center with 1.5 T General Electric Genesis Signa [23] and CT images with the $512 \times 512$ resolution are collected from OsiriX [24]. Table 2 presents test set II containing 16-bit depth CT and MR images of different resolutions obtained from the Cancer Imaging Archive [25-27]. Table 1 and Table 2 provide the details of images utilized in this research.

Table 1. Test set I composed of 8-bit depth CT and MR images of $256 \times 256$ and $512 \times 512$ resolutions

\begin{tabular}{|ccc|}
\hline Sequence Name & Image Size & Slices \\
\hline CT_Aperts & $256 \times 256$ & 97 \\
\hline CT_carotid & $256 \times 256$ & 74 \\
\hline CT_skull & $256 \times 256$ & 203 \\
\hline CT_wrist & $256 \times 256$ & 183 \\
\hline MR_liver_T1 & $256 \times 256$ & 58 \\
\hline MR_liver_T2e1 & $256 \times 256$ & 58 \\
\hline MR_ped_chest & $256 \times 256$ & 77 \\
\hline MR_sag_head & $256 \times 256$ & 58 \\
\hline MR-SAG-T1 & $512 \times 512$ & 13 \\
\hline MR-COR-T1 & $512 \times 512$ & 20 \\
\hline CT_BREBIX & $512 \times 512$ & 245 \\
\hline CT_MAGIX & $512 \times 512$ & 77 \\
\hline
\end{tabular}


Table 2. Test set II standard dataset composed of 16-bit depth CT and MR images of different resolutions

\begin{tabular}{|ccc|}
\hline Sequence & Image Size & Slices \\
\hline CT_Lung_R13 & $512 \times 512$ & 67 \\
\hline CT_Lung_R4 & $512 \times 512$ & 68 \\
\hline MR_Neuro & $256 \times 256$ & 176 \\
\hline MR_Breast & $288 \times 288$ & 60 \\
\hline
\end{tabular}

\subsection{PREDICTIVE CODING TECHNIQUE}

Adjacent pixels in 2D images are highly correlated to each other and this correlation is a measure of spatial redundancy. In the predictive coding technique, this spatial redundancy is removed by predictors by predicting the current pixel and obtaining a residual value. The difference between the predicted image and the original image gives a residual image and better prediction and results in a low correlation [28]. The efficiency of the predictor depends on the entropy of the residual image; the smaller the entropy, the better the prediction. The residual image has less entropy, hence a smaller number of bits is used to encode the residual image. Statistical redundancy present in the image can be removed by encoders after prediction. The efficiency of the encoder depends on how well it reduces the code length of the residual image. For lossless compression of an image, prediction and encoding are the two important steps that finally provide the compressed image. A general block diagram of the predictive coding technique is given in Figure 1.

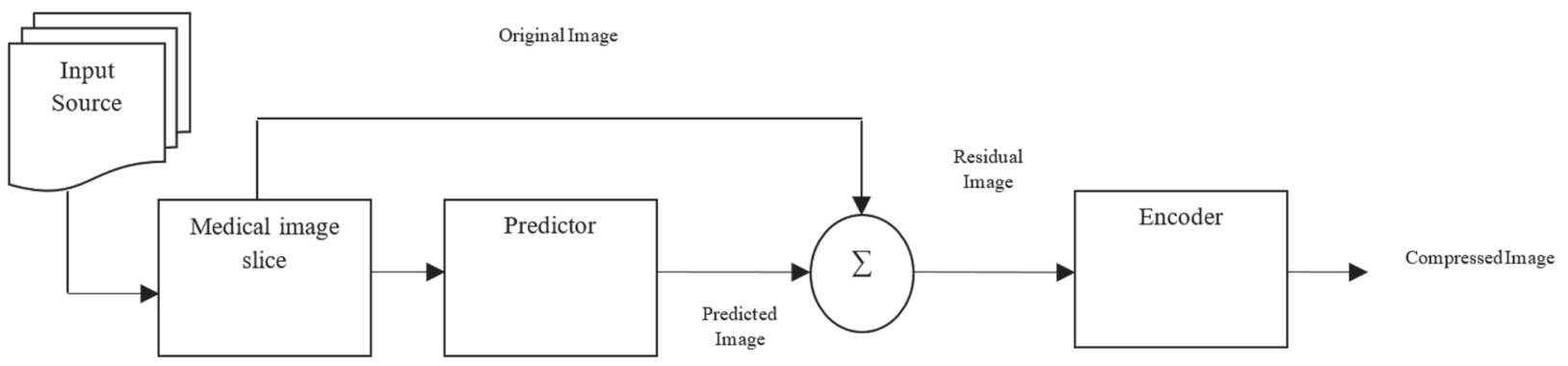

Fig. 1. Basic block diagram of the predictive coding technique

For compression of volumetric images, 2D predictors are operated individually for each image slice in the volumetric image sequence. Causal neighbours in the 2D image are commonly labelled as shown in equation 1.

$X_{N}=X[i, j-1], X_{w}=X[i-1, j]$,

$\mathrm{X}_{\mathrm{NW}}=\mathrm{X}[\mathrm{i}-1, \mathrm{j}-1], \mathrm{X}_{\mathrm{NE}}=\mathrm{X}[\mathrm{i}+1, \mathrm{j}-1]$

$\mathrm{X}_{\mathrm{NN}}=\mathrm{X}[\mathrm{i}, \mathrm{j}-2], \mathrm{X}_{\mathrm{NW}}=\mathrm{X}[\mathrm{i}-2, \mathrm{j}]$,

$\mathrm{X}_{\mathrm{NNE}}=\mathrm{X}[\mathrm{i}+1, \mathrm{j}-2]$

Where, $X_{i, j}=$ Current pixel to be predicted

$\mathrm{N}=$ North, $\mathrm{W}=$ West, $\mathrm{NW}=$ North West,

$\mathrm{NE}=$ North East, $\mathrm{NN}=$ North-North

$\mathrm{NW}=$ North West, $\mathrm{NNE}=$ North-North East

Standard 2D benchmark predictor algorithms: MED and GAP are used for prediction in the lossless predictive technique; MED is employed with the JPEG-LS standard algorithm and the MED predictor makes the JPEG-LS less computational complex but does not provide high efficiency. On the other hand, GAP is more efficient than MED, but it is computationally complex to implement. GAP adapts itself to the gradients of the predicted pixel's neighbour and it is a threshold based predictor with heuristic threshold values. A combination of both standard predictors results in GED that takes the merit of simplicity and efficiency from both MED and GAP predictors. GED is also threshold based, just like GAP, but the threshold value is user-defined in case of GED [15]. In literature, different encoding techniques are available like Huffman, run-length, Dictionary, arithmetic and bit-plane coding, etc. In this research, arithmetic coding is utilized as it is the most efficient coding technique among other encoding techniques [29] because fewer bits are used to encode frequently seen symbols and large numbers of bits are used for rarely seen symbols.

\subsection{PROPOSED METHODOLOGY}

This section presents the details of the proposed technique for lossless compression of medical images with varying image bit depth, resolution and modalities. The proposed technique involves two interdependent techniques, i.e. the resolution independent gradient edge detector and block adaptive arithmetic encoding detailed in this section.

\subsubsection{Resolution Independent Gradient Edge Detector (RIGED)}

The efficiency of the predictor depends on how effectively it can decrease the entropy of the residual image. Predictor efficiency can be improved by making it modality and resolution independent. The proposed RIGED is both modality and resolution independent. RIGED is an extension of the GED predictor that is the best combination of a simple algorithm and efficient performance in terms of entropy. The existing GED is threshold based, that is user-defined and there is no particular method to select the threshold for prediction [30]. 
For perfect prediction, a particular threshold value should be selected so that the entropy of the residual image is minimized. The proposed RIGED is designed by selecting a common universal threshold that is optimal for different image resolutions and modalities. RIGED is also designed to deal with high bit depth images up to 16-bit depth because advanced image scanning techniques produce higher bit depth images of large resolution. The threshold level for prediction of different bit depth medical images can be up to $2^{\text {bit depth }}$

Spatial or inter-pixel redundancy present in an image can be removed by predictors from the image. A common scheme for labelling causal neighbours in 2D predictors is shown in Figure 2.

\begin{tabular}{l|c|c|c|l} 
& & NN & NNE & \\
\hline & NW & N & NE & \\
\hline$W W$ & $W$ & $x_{i, j}$ & & \\
\hline & & & &
\end{tabular}

Fig. 2. Common scheme of causal template for labelling neighbours

Referring to this causal template, the algorithm of RIGED is proposed as shown in equation 2 .

Vertical gradient: $A_{v}=|N W-W|+|N N-N|$

Horizontal gradient: $A_{h}=|W W-W|+|N W-N|$

if $A_{v}-A_{h}>T$, then $P_{x}=W$;

if the difference of vertical and horizontal gradients is greater than a threshold,then the predicted pixel direction is west.

else if $A_{v}-A_{h}<-T, P X=N$;

if the difference of vertical and horizontal gradients is less than a threshold,then the predicted pixel direction is north,

else $\mathrm{P}_{\mathrm{x}}=\mathrm{N}+\mathrm{W}-\mathrm{NW}$,

where $\mathrm{T}=$ Threshold and $\mathrm{Av}$ and $\mathrm{Ah}$ are Vertical and Horizontal Gradients

$\mathrm{T}=44$ (Common threshold for every modality and resolution of 8-bit depth medical image)

$\mathrm{T}=768$ (Common threshold for every modality and resolution of 16-bit depth medical image)

$T=2^{5}$ (32) specifically for resolution $256 \times 256$

and 8-bit depth image

$\mathrm{T}=2^{6}(64)$ specifically for resolution $512 \times 512$

and 8-bit depth image

$\mathrm{T}=2^{9}(512)$ specifically for resolution $256 \times 256$ and 16-bit depth image

$\mathrm{T}=2^{10}$ (1024) specifically for resolution $512 \times 512$

and 16-bit depth imag
With proper selection of the threshold value for prediction, RIGED provides perfect prediction with a low entropy residual image.

\subsubsection{Block Adaptive Arithmetic Encoding (BAAE)}

Encoding is the second major step in the predictive coding technique to encode the residual image that provides compression in terms of BPP. The residual image obtained by the proposed RIGED is divided into non-overlapping blocks before arithmetic encoding because there are different error probabilities in different image regions. Image segmentation is done in different block sizes ranging from $4 \times 4$ to $128 \times 128$ as shown in Figure 3. Blocks are classified into different groups on the basis of mean absolute error. Each group is encoded separately by the arithmetic encoder.

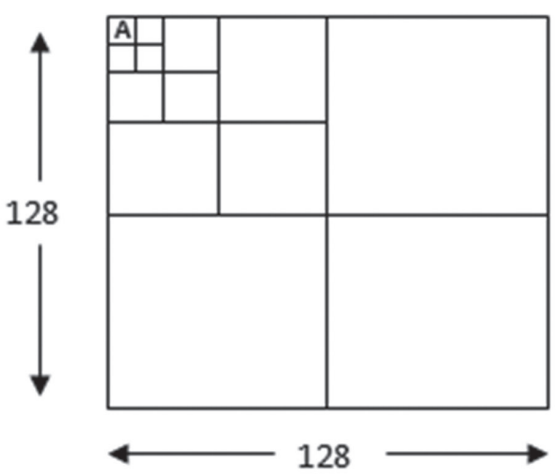

(a)

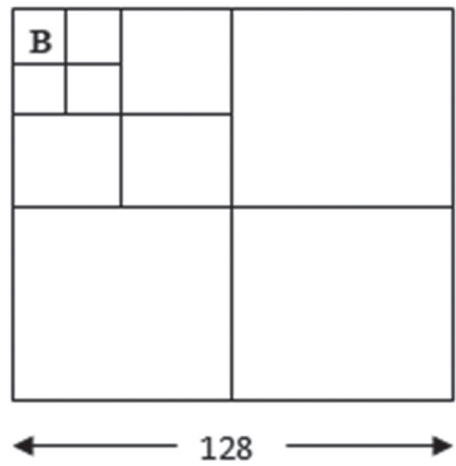

(b)

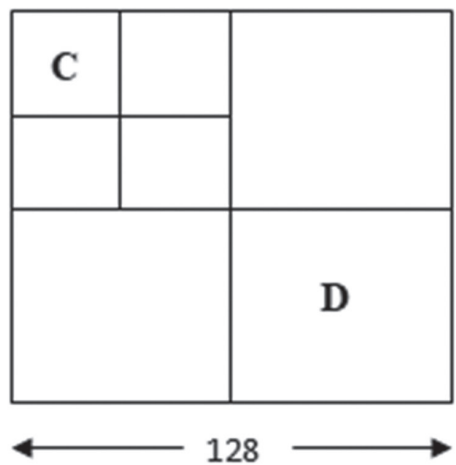

(c)

Fig. 3. Adaptive blocks with different sizes.

(a) The size of block ' $A$ ' is $8 \times 8$.

(b) The size of block ' $B$ ' is $16 \times 16$.

(c) The sizes of block ' $C$ ' and block ' $\mathrm{D}$ ' are $32 \times 32$ and $64 \times 64$, respectively. 
Empirical analysis is done for different block sizes and finally an optimal block size is selected after calculating the BPP values for the complete dataset. Overhead is important at the decoder side to decompress the image. Calculated overhead provides the side information of blocks. This overhead information is also considered for overall BPP calculation of the encoded residual image. The overall BPP is calculated by adding the overhead information and bit-stream of the encoded residual image.

\subsubsection{Architecture of BAAE Employing RIGED Predictor for 8-bit and 16-bit Depth Images}

The architecture of the proposed system is shown in Figure 4.

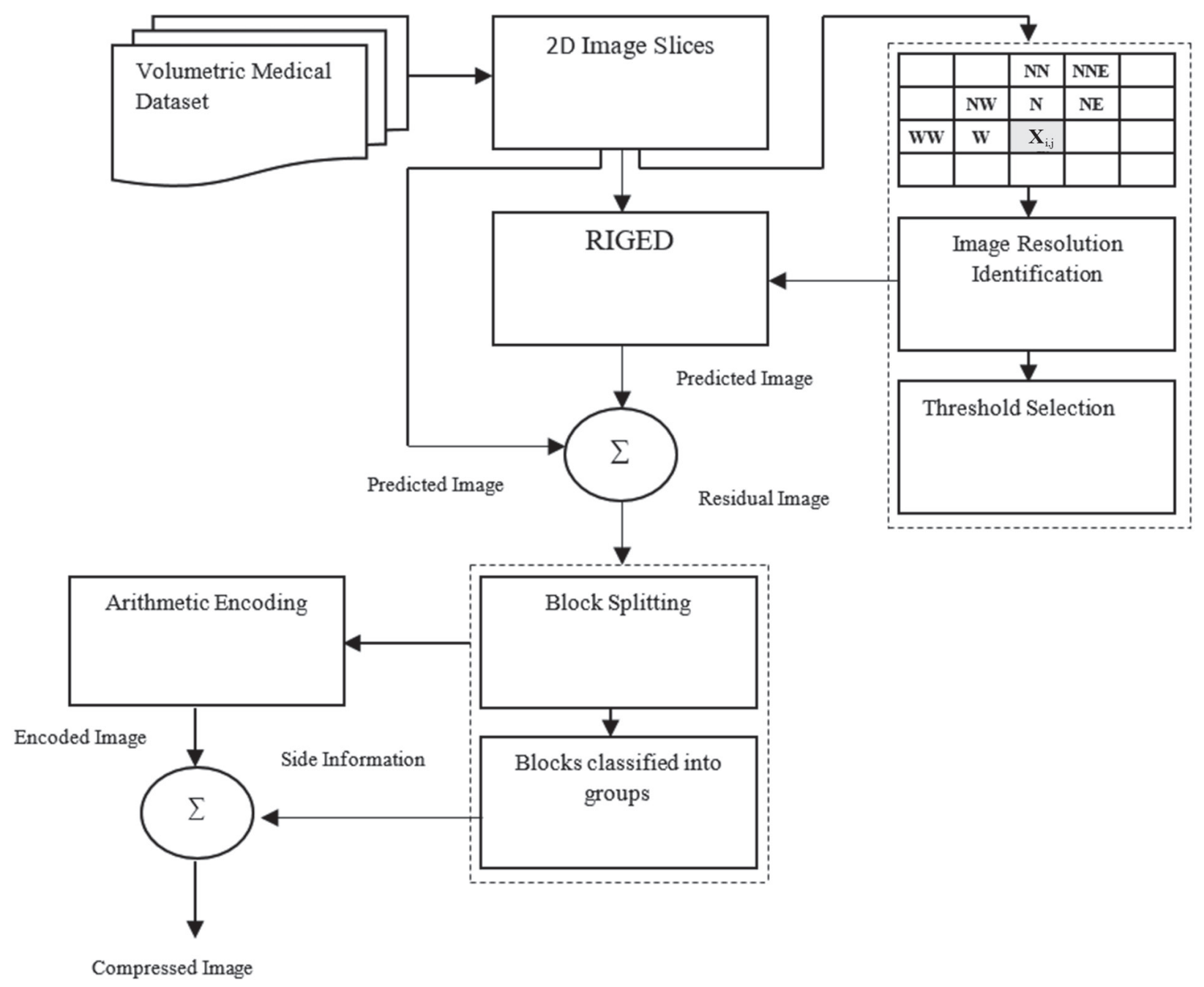

Fig. 4. Proposed flow chart for the block adaptive arithmetic encoding technique employing RIGED

The proposed algorithm is a common solution for compression of different types of medical images with varying resolutions, modalities and bit depth. The proposed BAAE technique employing RIGED is a 2D compression process applied to volumetric medical images containing image sequences on a frame-by-frame basis. Each frame is predicted by RIGED and by subtracting the predicted image slice from the original image we obtain the residual image with a lower entropy as compared to the original image slice. A lower entropy residual image is divided into optimal size blocks and blocks are grouped on the basis of the average of absolute error. Further different groups are separately entropy-encoded by the arithmetic encoder to achieve a compressed image. For lossless compression of a volumetric dataset, the overall procedure is repeated for the next image slices and a weighted average is calculated for the complete image set. The proposed architecture is not only applicable to low bit depth medical dataset but also to high bit depth (16 bits/pixel) medical images.

Performance of the proposed technique is evaluated by entropy and BPP values. Entropy is calculated after RIGED prediction and the overall compression performance in terms of BPP is calculated after block-based encoding. Entropy describes the total number of bits required to represent image information [31]. The over- 
all compression performance is calculated and the data size of a compressed image depends on BPP and image resolution. A smaller number of bits required to store the compressed image shows a high compression efficiency.

The compression ratio $(\mathrm{CR})$ is inversely related to BPP values [32], [33].

\section{RESULTS AND DISCUSSION}

In this section, performance of the proposed algorithm on medical images of varying bit depth, modalities and image resolutions collected from different sources is validated and discussed in terms of entropy and BPP values. The effect of an optimal threshold value for prediction and effect on the BPP value when the residual image is segmented into non-overlapping blocks before entropy encoding is observed for 8-bit and 16-bit depth images. A comparison of the proposed algorithm is done with the results obtained for standard CALIC and JPEG-LS lossless compression techniques.

\subsection{PERFORMANCE ASSESSMENT OF THE RIGED ALGORITHM}

Experiments were performed to test the proposed RIGED performance on a number of medical image slices of different modalities. Parameter assessment obtained after the RIGED predictor on prediction performance is analyzed for 8 and 16-bit depth images. The evaluated parameter is entropy at different threshold values for both test sets given in Section 2. The purpose of the proposed RIGED is to make the GED algorithm resolution and modality independent. The entropy of the original image and entropy values obtained for the residual image predicted at different thresholds for test set I ( 8 bits/pixel) are given in Table 3. A weighted average is evaluated for the complete dataset on the basis of the number of slices in every modality.

Table 3. Entropy obtained by the GED predictor for 8-bit depth images at different T-levels on the basis of $2^{n}$

\begin{tabular}{cccccccc}
\hline Test Set I & $\begin{array}{c}\text { Original } \\
\text { Entropy }\end{array}$ & $\mathbf{8}$ & $\mathbf{1 6}$ & $\mathbf{3 2}$ & $\mathbf{6 4}$ & $\mathbf{1 2 8}$ & $\mathbf{2 5 6}$ \\
\hline CT_Aperts & 2.7471 & 1.6423 & 1.6370 & 1.6345 & 1.6459 & 1.6534 & 1.6534 \\
\hline CT_carotid & 3.9375 & 2.5570 & 2.5481 & 2.5521 & 2.5694 & 2.5772 & 2.5772 \\
\hline CT_skull & 4.5983 & 3.6242 & 3.6171 & 3.6137 & 3.6251 & 3.6481 & 3.6525 \\
\hline CT_wrist & 4.4068 & 2.2856 & 2.2551 & 2.2204 & 2.2323 & 2.2441 & 2.2466 \\
\hline MR_liver_t1 & 4.9023 & 3.4716 & 3.4607 & 3.4493 & 3.4520 & 3.4513 & 3.4511 \\
\hline MR_liver_t2e1 & 6.4190 & 3.7210 & 3.6962 & 3.6780 & 3.6805 & 3.6806 & 3.6807 \\
\hline MR_ped_chest & 4.8892 & 3.3472 & 3.3360 & 3.3284 & 3.3306 & 3.3406 & 3.3430 \\
\hline MR_sag_head & 4.3164 & 3.6042 & 3.6234 & 3.6397 & 3.6524 & 3.6591 & 3.6591 \\
\hline MR-SAG-T1 & 5.5833 & 3.3170 & 3.2969 & 3.2798 & 3.2736 & 3.2770 & 3.2784 \\
\hline MR-COR-T1 & 5.6467 & 3.4986 & 3.4610 & 3.4279 & 3.4091 & 3.4259 & 3.4270 \\
\hline CT_BREBIX & 3.6900 & 2.8345 & 2.8373 & 2.8495 & 2.8390 & 2.8740 & 2.8872 \\
\hline CT_MAGIX & 4.8084 & 2.4503 & 2.3745 & 2.3334 & 2.3196 & 2.3283 & 2.3292 \\
\hline Weighted & $\mathbf{4 . 2 2 8 3}$ & $\mathbf{2 . 8 6 0 7}$ & $\mathbf{2 . 8 4 5 1}$ & $\mathbf{2 . 8 3 9 4}$ & $\mathbf{2 . 8 3 6 8}$ & $\mathbf{2 . 8 5 7 1}$ & $\mathbf{2 . 8 6 3 7}$ \\
Average & & & & & & & \\
\hline
\end{tabular}

Prediction performance observed from the results shown in Table 3 represent a significant variation in entropy values at different threshold $(T)$ levels. The entropy value at $2^{3}$ is highest of the value 2.8607 and it decreases for a higher threshold level. There is a minor variation in the entropy value when T-level varies from $2^{5}$ to $2^{6}$ quantified as 2.8394 to 2.8368 , but increases after point $2^{6}$ up to $2^{8}$ for 8 -bit depth images. As minimum entropy values are obtained between $2^{5}$ to $2^{6}$, so T-levels after $2^{5}$ are tested in the small difference of threshold value 16 . The weighted average calculated for test set I at T-levels from $2^{5}$ to $2^{7}$ in the gap of 16 is shown in Figure 5.

It is clear from Figure 3 that there is a significant variation when the threshold value changes from 32 to 48 as ranging from 2.8394 to 2.83574 , and after 48 , entropy starts increasing. Threshold 48 provides minimum entropy as shown in Figure 5, and deep analysis is done before and after 48 in the difference of 1 for the exact threshold point as depicted in Figure 6. For a clear distinction, the figure shows the weighted average value 
of entropy for the complete set of 8-bit depth images with a varying number of slices.

After testing different threshold levels from 43 to 53 for the minimum entropy value, it is observed that the minimum entropy value 2.83528 is obtained at the value of 44 . There is a minor variation at the threshold values of 43 and 44 and after 44 it starts increasing. After empirical experimental and extensive analysis of the threshold value for prediction to achieve minimum entropy of the residual, the threshold value of 44 came with the lowest entropy.

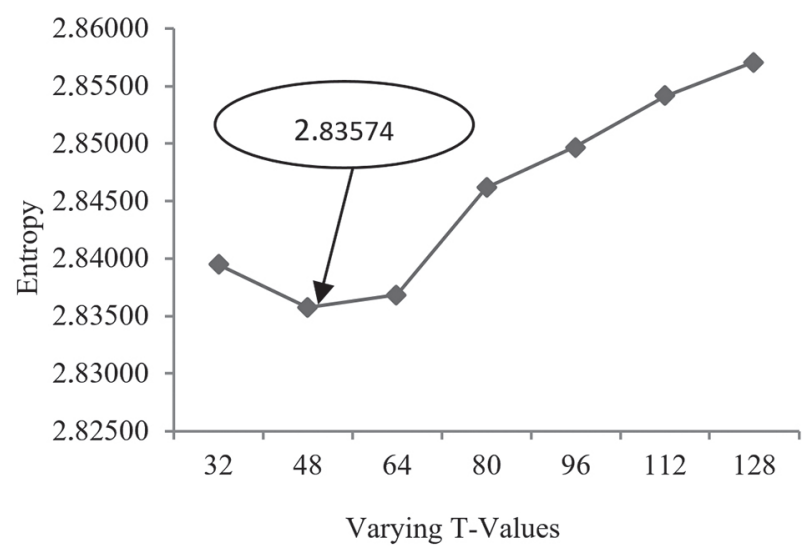

Fig. 5. Entropy obtained by the GED predictor for 8-bit depth images at different threshold values in difference of 16

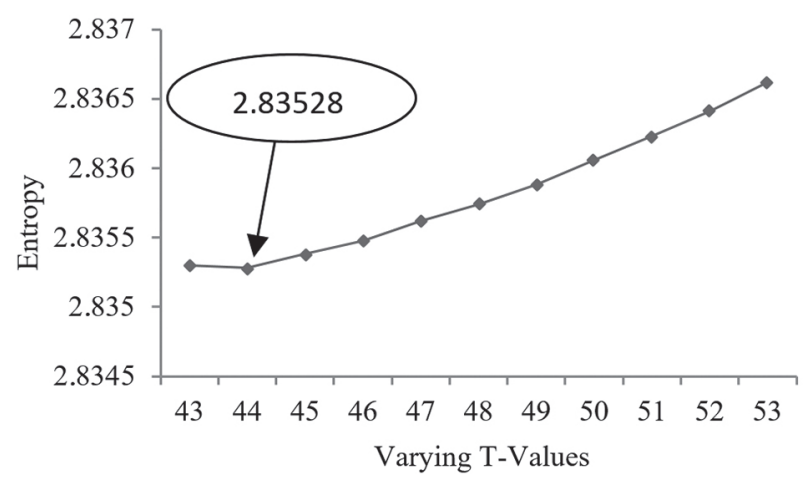

Fig. 6. Entropy obtained by the GED predictor for 8-bit depth images at different threshold values in difference of 1

\subsection{EXTENSION OF RIGED FOR 16-BIT DEPTH MEDICAL IMAGES}

Designed for 8-bit depth medical images, RIGED is also extended for 16-bit depth because most of the images present higher bit depth up to 16 bits. The threshold value is selected for 16-bit depth images ranging up to 216. A common universal threshold obtained for 16-bit depth images is independent of image modality and resolution and different from the value attained for 8-bit depth images. Figure 7 presents prediction results in terms of entropy for test set II.

Entropy values of the residual image at different Tlevels on the range of $2^{n}$ as shown in Figure 5 shows that the entropy value is high at lower threshold values and with an increase in the threshold level for prediction, the entropy value starts decreasing. Entropy at the threshold value of $2^{3}$ is 7.02412 and after this point of the threshold, it decreases up to the T-level of $2^{10}$ (1024) and reaches 6.5871. Minimum entropy values are found between $2^{9}$ and $2^{10}$, so for an optimal threshold value, RIGED is tested on different threshold values ranging between $2^{9}$ and $2^{10}$ at the difference of 128 . The weighted average of entropy values for complete test set II is calculated for threshold values between $2^{9}$ and $2^{10}$ i.e., at 512, 640, 768, 89 and 1024.

Entropy obtained at varying threshold values for test set II are presented in Table 4, and a common threshold is selected that is independent of image modality and resolution of 16-bit depth images providing minimum entropy.

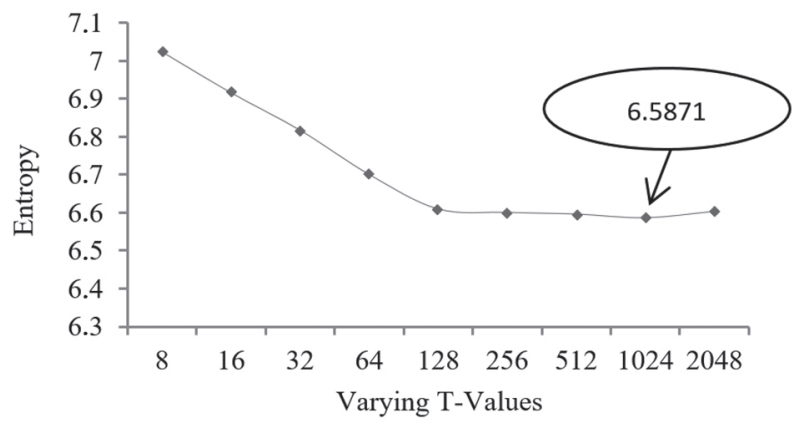

Fig. 7. Entropy obtained by the GED predictor for 16-bit depth images at 2n T-Levels

\begin{tabular}{ccccccc}
\hline Test Set II & $\begin{array}{c}\text { Original } \\
\text { Entropy }\end{array}$ & $\mathbf{5 1 2}$ & $\mathbf{6 4 0}$ & $\mathbf{7 6 8}$ & $\mathbf{8 9 6}$ & $\mathbf{1 0 2 4}$ \\
\hline CT_Lung & 9.1207 & 6.0923 & 6.0837 & 6.0813 & 6.0816 & 6.0828 \\
\hline CT-Lung & 7.9619 & 7.0347 & 7.0259 & 7.0022 & 7.0091 & 7.0127 \\
\hline MR_Breast & 8.7692 & 7.1633 & 7.1508 & 7.1483 & 7.1533 & 7.1643 \\
\hline MR_Neuro & 7.1152 & 6.4453 & 6.4444 & 6.4439 & 6.4461 & 6.4484 \\
\hline $\begin{array}{c}\text { Weighted } \\
\text { Average }\end{array}$ & $\mathbf{8 . 2 4 3 3}$ & $\mathbf{6 . 5 9 6 8}$ & $\mathbf{6 . 5 8 9 8}$ & $\mathbf{6 . 5 8 0 8}$ & $\mathbf{6 . 5 8 3 9}$ & $\mathbf{6 . 5 8 7 1}$ \\
\hline
\end{tabular}


Results given in Table 4 show that the entropy value obtained at the threshold value of 512 is maximal and it starts decreasing by the threshold value of 768 . The threshold value of 768 came up with a minimum entropy value for 16-bit depth medical images and is independent of image resolution and modality.

\subsection{PERFORMANCE EVALUATION OF BAAE}

T-values of 44 and 768 are selected pursuant to the proposed RIGED algorithm for prediction. After RIGED prediction of 8-bit and 16-bit depth images, the residual image is divided into different block sizes before encoding. The BPP value is obtained after arithmetic encoding for complete datasets given in Section 2 for different block sizes ranging from $4 \times 4$ to $128 \times 128$. The average of BPP values based on the number of slices is calculated and presented in tables 5 and 6 for 8-bit and 16-bit depth images, respectively.

It is clear from the results shown in Table 5 that the compression performance shows a significant variation for a different number of block sizes. The overall BPP values vary with varying block sizes when overhead is also considered. After experimental analysis on different block sizes for BPP calculation, the best block size which finally provides the minimum BPP value is selected.

While considering overhead with the compressed image, the weighted average of the BPP value at the smallest block size of $4 \times 4$ is 2.50 and 6.05 for 8 -bit and 16-bit, respectively. After $4 \times 4, \mathrm{BPP}$ values decrease to 2.31 for test set I and to 5.29 for test set II at $8 \times 8$. For a higher block size after $8 \times 8$, overall BPP value continue increasing for both medical test sets. This is due to overhead information; overhead is large and code length is small for a smaller block size. The implication drawn from the obtained results is that when code length of a compressed image is combined with the BPP overhead, then the minimum overall BPP value is achieved at an $8 \times 8$ block size. The BPP values obtained from the proposed technique show the same results for different types of images contained in test set I and test set II. So, proposed BAAE employing RIGED gives optimal performance evaluation in the form of BPP, which is independent of modality and resolution used.

Table 5. Compression results (in BPP) for a varying number of block size from the proposed technique for 8-bit depth images

\begin{tabular}{ccccccc}
\hline Test Set I & \multicolumn{7}{c}{ BPP for different block sizes } \\
& $\mathbf{4 \times 4}$ & $\mathbf{8 \times 8}$ & $\mathbf{1 6 \times 1 6}$ & $\mathbf{3 2 \times 3 2}$ & $\mathbf{6 4 \times 6 4}$ & $\mathbf{1 2 8 \times 1 2 8}$ \\
\hline CT_Aperts & 1.09 & 1.01 & 1.07 & 1.10 & 1.12 & 1.15 \\
\hline CT_carotid & 1.69 & 1.67 & 2.08 & 2.13 & 2.15 & 2.18 \\
\hline CT_skull & 2.88 & 2.60 & 2.87 & 2.91 & 2.93 & 3.01 \\
\hline CT_wrist & 1.97 & 1.54 & 1.64 & 1.69 & 1.73 & 1.76 \\
\hline MR_liver_t1 & 3.38 & 3.02 & 3.21 & 3.22 & 3.24 & 3.25 \\
\hline MR_liver_t2e1 & 2.55 & 2.26 & 2.29 & 2.32 & 2.35 & 2.37 \\
\hline MR_ped_chest & 2.71 & 2.60 & 2.62 & 2.67 & 2.71 & 2.73 \\
\hline MR_sag_head & 2.75 & 2.50 & 2.52 & 2.56 & 2.58 & 2.62 \\
\hline MR-SAG-T1 & 2.55 & 2.78 & 2.88 & 2.92 & 3.01 & 3.04 \\
\hline MR-COR-T1 & 2.93 & 2.71 & 2.94 & 2.95 & 2.96 & 2.97 \\
\hline CT_BREBIX & 2.60 & 2.45 & 2.63 & 2.66 & 2.71 & 2.76 \\
\hline CT_MAGIX & 2.47 & 2.26 & 2.31 & 2.34 & 2.45 & 2.49 \\
\hline Weighted Average & $\mathbf{2 . 5 0}$ & $\mathbf{2 . 3 1}$ & $\mathbf{2 . 4 6}$ & $\mathbf{2 . 4 9}$ & $\mathbf{2 . 5 4}$ & $\mathbf{2 . 5 9}$ \\
\hline
\end{tabular}

Table 6. Compression results (in BPP) for varying number of block size from proposed technique for 16-bit depth images

\begin{tabular}{cccccccc}
\hline & & \multicolumn{5}{c}{ BPP for different block sizes } \\
Test Set II & $\mathbf{4 \times 4}$ & $\mathbf{8 \times 8}$ & $\mathbf{1 6 \times 1 6}$ & $\mathbf{3 2 \times 3 2}$ & $\mathbf{6 4 \times 6 4}$ & $\mathbf{1 2 8 \times 1 2 8}$ \\
\hline CT_Lung & 6.10 & 5.40 & 5.42 & 5.95 & 6.00 & 6.05 \\
CT-Lung & 6.55 & 5.63 & 5.63 & 6.27 & 6.37 & 6.55 \\
MR_Breast & 5.61 & 5.08 & 5.10 & 5.52 & 5.75 & 5.93 \\
MR_Neuro & 4.77 & 3.90 & 3.93 & 4.14 & 4.36 & 4.58 \\
\hline Weighted Average & $\mathbf{6 . 0 5}$ & $\mathbf{5 . 2 9}$ & $\mathbf{5 . 3 1}$ & $\mathbf{5 . 8 2}$ & $\mathbf{5 . 9 4}$ & $\mathbf{6 . 0 9}$
\end{tabular}


Comparative analysis of both cases, with and without overhead consideration with code length of the compressed image for the calculation of compression in terms of BPP, is depicted in Figure 8.

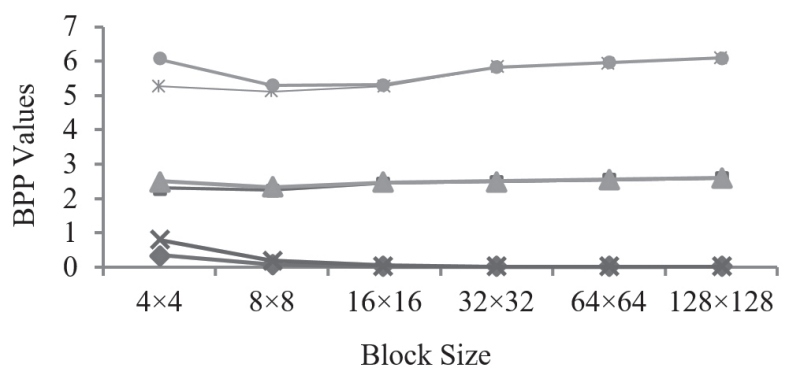

$$
\begin{aligned}
& \longrightarrow 8 \text { Overhead } \rightarrow-8 \text { Without Overhead } \\
& \simeq 8 \text { Overall } \quad \simeq 16 \text { Overhead } \\
& \text { * 16 Without Overhead } \multimap 16 \text { Overall }
\end{aligned}
$$

Fig. 8. Variation of the weighted average of BPP values with and without overhead for 8 and 16-bit images at different block sizes

It can be concluded from Fig 6 that when overhead is not considered with code length, then the minimum BPP value is obtained at $4 \times 4$ and it continues increasing for larger block sizes. When overhead is also considered with code length of the compressed image, then the minimum BPP value is obtained at $8 \times 8$. This is be- cause code length increases and overhead decreases after $8 \times 8$, so when overhead and code length of the image are combined, then the minimum value of the overall BPP is obtained at $8 \times 8$ for both 8 -bit and 16bit depth images. There is a significant difference in BPP values at $4 \times 4$, with overhead considered or not with code length of the compressed image. At $8 \times 8$ and $16 \times 16$, there is a minor variation in BPP values and for a larger block size after $16 \times 16$, BPP values for both cases are almost equal because overhead BPP is very small for larger block sizes.

\subsection{COMPARISON OF THE PROPOSED TECHNIQUE WITH LOSSLESS COMPRESSION STANDARDS CALIC AND JPEG-LS}

In this subsection, the performance of the proposed $B A A E+R I G E D$ technique is analysed by comparing compression results in terms of BPP with state-of-the art CALIC and JPEG-LS algorithms reported in the literature as shown in Table 7. Compression results (in BPP) using JPEG-LS and CALIC for MR and CT images of resolution $256 \times 256$ are provided by M.J Weinberger et al. [14] and CALIC Implementation [34]. BPP values obtained for MR images having resolution $512 \times 512$ are provided by E. Puthooran et al. [32] using standard JPEG-LS and CALIC. Compression results obtained by standard JPEG-LS and CALIC for test set II are obtained by Lucas et al. [35].

Table 7. Comparison of compression performance (in BPP) by using the proposed technique and the existing lossless coding methods for both test sets

\begin{tabular}{cccc}
\hline Image Modality Sets & & Coding Techniques & \\
& CALIC & JPEG-LS & Proposed Technique \\
\hline CT_Aperts & 1.00 & 1.06 & 1.01 \\
\hline CT_carotid & 1.68 & 1.78 & 2.68 \\
\hline CT_skull & 2.63 & 2.76 & 1.54 \\
\hline CT_wrist & 1.55 & 1.63 & 3.03 \\
\hline MR_liver_t1 & 3.02 & 3.16 & 2.27 \\
\hline MR_liver_t2e1 & 2.27 & 2.42 & 2.61 \\
\hline MR_ped_chest & 2.79 & 2.94 & 2.50 \\
\hline MR_sag_head & 2.52 & 2.58 & 2.79 \\
\hline MR_SAG-T1 & 2.70 & 2.98 & 2.71 \\
\hline MR_COR-T1 & 2.51 & 2.72 & 5.40 \\
\hline CT_Lung & 5.44 & 5.66 & 5.63 \\
\hline CT-Lung & 5.81 & 5.80 & 5.08 \\
\hline MR_Neuro & 5.12 & 5.30 & 3.91 \\
\hline MR_Breast & 4.11 & 3.93 & 3.59 \\
\hline Weighted Average & 3.64 & 3.75 & \\
\hline
\end{tabular}

The weighted average of the BPP value 3.59 is obtained by the proposed BAAE+RIGED method and 3.64 and 3.75 of BPP values are obtained by CALIC and JPEG-LS, respectively, for the complete medical test set reported in Section 2. Compression results in terms of BPP obtained by the proposed technique and standard techniques for a sequence of images are processed individually. The compression result of the 
proposed BAAE+RIGED technique is better than standard techniques and the weighted average of percentage improvement is $0.70 \%$ and $4.62 \%$ over CALIC and JPEG-LS, respectively. Selection of an optimal threshold value to remove spatial redundancy and selection of an optimal block size to remove coding redundancy improves the compression performance of the proposed lossless coding technique.

The proposed algorithm is also compared with some other lossless coding techniques for a 16-bit depth dataset. The graphical representation shows percentage improvement of the proposed approach over other techniques like CALIC, JPEG-LS, JPEG-2000, (JP3D) and High Efficiency Video Coding (HEVC) [35], [36] for test set II in Figure 9.

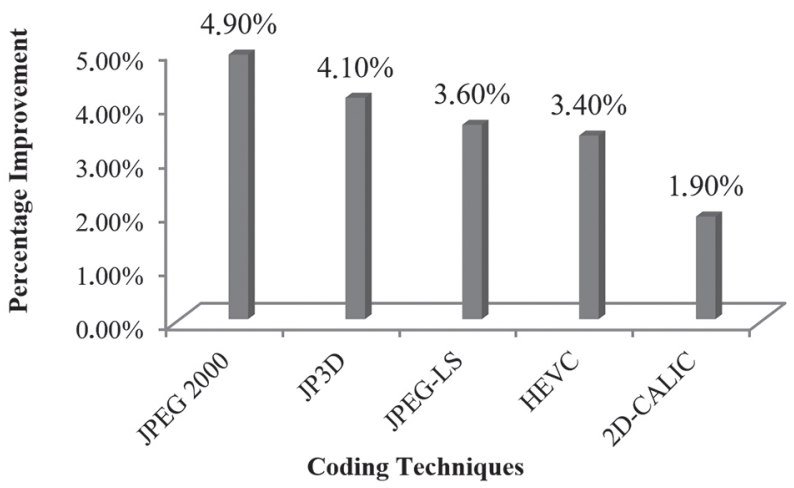

Fig. 9. Percentage improvement by the proposed technique over existing compression methods for test set II

It is observed that the performance of the proposed $\mathrm{BAAE}+\mathrm{RIGED}$ is better than with other lossless coding techniques in terms of BPP. BAAE+RIGED perform 1.90 $\%$ and $3.65 \%$ better than standard CALIC and JPEG-LS, respectively. $4.99 \%$ and $4.18 \%$ of compression gain is achieved over JPEG-2000 and its 3D extension (JP3D). A comparison is also made with other coding techniques such as M-CALIC, 3D-CALIC and HEVC to analyze the performance of the proposed technique. The proposed technique is $8.64 \%, 5.52 \%$ and $3.47 \%$ better than $\mathrm{M}$ CALIC, 3D-CALIC and HEVC, respectively. Improvement in compression performance is due to optimal threshold prediction value and optimal block size selection for removing interpixel redundancy and coding redundancy.

\section{CONCLUSION}

In this paper, an efficient lossless predictive algorithm is proposed for compression of volumetric medical images of 8-bit and 16-bit depth. The proposed algorithm is based on an optimal threshold value for prediction and an optimal block size for encoding a residual image. The proposed technique combines the RIGED predictor and BAAE to achieve efficient compression results in terms of BPP and is resolution and modality independent. Superiority of the proposed technique is compared with standard techniques and our extensive experimental results demonstrate that the proposed BAAE+RIGED provides better compression results than benchmark lossless compression algorithms JPEG-LS and CALIC. The proposed technique achieves percentage improvement of $0.70 \%$ and $4.62 \%$ for complete medical test sets over CALIC and JPEG-LS, respectively.

\section{REFERENCES}

[1] L. Koller, "The Evolution of Medical Imaging Technologies: Electric Meat and Physician's Shifting Gaze", EA-Journal, Vol. 2, No. 3, 2011, pp. 1-22.

[2] C-T Yanga, C-H Chen, M.-F. Yang, "Implementation of a medical image files accessing system in co-allocation data grids", Future Generation Computer Systems, Vol. 26, No. 8, 2010, pp. 1127-1140.

[3] U. Ji, "Effectiveness of Reconstruction Methods in Compressive Sensing for Biomedical Images", Journal of Global Pharma Technology, Vol. 6, No. 9, 2017, pp. 134-143.

[4] M. Singh, S. Kumar, S. Singh, M. Shrivastava, "Various Image Compression Techniques: Lossy and Lossless", International Journal of Computer Applications, Vol. 142, No. 6, 2016, pp. 23-26.

[5] R. Gupta, M. Kumar, R. Bathla, “Data Compression Lossless and Lossy Techniques", International Journal of Application or Innovation in Engineering \& Management, Vol. 5, No. 7, 2016, pp. 120-125.

[6] J. Reichel, G. Menegaz, M. J. Nadenau, M. Kunt, "Integer wavelet transform for embedded lossy to lossless image compression", IEEE Transactions on Image Processing, Vol. 10, No. 3, 2001 pp. 383-392.

[7] J. Puate, F. D. Jordan, "Using fractal compression scheme to embed a digital signature into an image", Proceedings of the SPIE Photonics East'96 Symposium, Boston, USA, 1996.

[8] El-Jaroudi, "Linear predictive coding", Wiley Encyclopedia of Telecommunications, 2003.

[9] D. O'Shaughnessy, "Linear predictive coding", IEEE Potentials, Vol. 7, No. 1, 1988, pp. 29-32.

[10] W. Pennebaker, J. Mitchel, "JPEG: Still Image Data Compression Standard", $1^{\text {st }}$ ed., Kluwer, 1992.

[11] ISO, International Standard, and IEC JTC, "Information technology-JPEG 2000 image coding systemPart 1", Core coding system. ISO/IEC 15444-1, 2001.

[12] D. Taubman, M. Marcelin, "JPEG2000: Image Compression Fundamentals, Standards and Practice", $2^{\text {nd }}$ ed., Kluwer, 2001. 
[13] X. Wu, N. Memon, "Context-based, adaptive, lossless image coding", IEEE Transactions on Communications, Vol. 45, No. 4, 1997, pp. 437-444.

[14] M. J. Weinberger, G. Seroussi, G. Sapiro, "The LOCO-I lossless image compression algorithm: principles and standardization into JPEG-LS", IEEE Transactions on Image Processing, Vol. 9, No. 8, 2000, pp. 1309-1324.

[15] A. Avramović, S. Savić, "Lossless predictive compression of medical images", Serbian Journal of Electrical Engineering, Vol. 8, No. 1, 2011, pp. 27-36.

[16] X. O. Zhao, Z. H. He, "Lossless image compression using super-spatial structure prediction", IEEE Signal Processing Letters, Vol. 17, No. 4, 2010, pp. 383-386.

[17] G. Al-Khafaji, H. Al-Mahmood, "Lossless Compression of Medical Images using Multiresolution Polynomial Approximation Model", International Journal of Computer Applications, Vol. 76, No. 3, 2013, pp. 38-42.

[18] R. Jovanovic, R. A. Lorentz, "Adaptive lossless prediction based image compression", Applied Mathematics \& Information Sciences, Vol. 8, No. 1, 2014, pp. 153-160.

[19] X. Song, Q. Huang, S. Chang, J. He, H. Wang, “Novel near-lossless compression algorithm for medical sequence images with adaptive block-based spatial prediction", Journal of Digital Imaging, Vol. 29, No. 6, 2016, pp. 706-715.

[20] X, Song, Q. Huang, S. Chang, J. He, H. Wang, “Lossless medical image compression using geometryadaptive partitioning and least square-based prediction", Medical \& biological engineering \& computing, Vol. 56, No. 6, 2018, pp. 957-966.

[21] A. Avramović, B. Reljin, "Gradient edge detection predictor for image lossless compression" Proceedings of the 52nd International Symposium ELMAR-2010, Zadar, Croatia, 15-17 September 2010, pp. 131-134.

[22] CIPR, http://www.cipr.rpi.edu/resource/sequences/sequence01.html (accessed: 2018)

[23] Micro-Dicom Dataset, http://www.microdicom. com/downloads.html (accessed: 2018)

[24] OsiriX Dataset, http://pubimage.hcuge.ch:8080/ (accessed: 2018)

[25] Grove et al., "Data from: Quantitative computed tomographic descriptors associate tumor shape complexity and intratumor heterogeneity with prognosis in lung adenocarcinoma", The Cancer
Imaging Archive, 2015, http://doi.org/10.7937/ K9/TCIA.2015.A6V7JIWX (accessed: 2018)

[26] Data from RIDER NEURO MRI, The Cancer Imaging Archive, 2015, http://dx.doi.org/10.7937/K9/TCIA. 2015.VOSN3HN1 (accessed: 2018)

[27] Meyer et al., "Data from RIDER Breast MRI", The Cancer Imaging Archive, 2015, http://doi. org/10.7937/K9/TCIA. 2015.H1SXNUXL (accessed: 2018)

[28] D. Shuai, L. Huang, P. Zhang, "Predictive Coding of Lossless Data Compression: A New Particle Dynamics Model", Proceeding of the IEEE International Symposium on Industrial Electronics, Montreal, Canada, 9-13 July 2006, pp. 565-570.

[29] G. A. Triantafyllidis, M. G. Strintzis, "A context based adaptive arithmetic coding technique for lossless image compression", IEEE Signal Processing Letters, Vol. 6, No. 7, 1999, pp. 168-170.

[30] Urvashi, E. Puthooran, M. Sood, "Implementation and Performance Assessment of Gradient Edge Detection Predictor for Reversible Compression of Biomedical Images", Proceedings of the International Conference on Advanced Informatics for Computing Research, Shimla, India, 14-15 July 2018, pp. 195-205.

[31] S. Shridevi, V. R., Vijaykumar, Anuja, "A Survey on Various Compression Methods for Medical Images", International Journal of Intelligent System and Applications, Vol. 3, 2012, pp. 13-19.

[32] E. Puthooran, R. S. Anand, S. Mukherjee, “Lossless compression of medical images using a dual level DPCM with context adaptive switching neural network predictor", International Journal of Computational Intelligence Systems, Vol.6, No. 6, 2013, pp.1082-1093.

[33] C. Bhardwaj, "Implementation and Performance Assessment of Compressed Sensing for Images and Video Signals", Journal of Global Pharma Technology, Vol. 6, No. 9, 2017, pp. 123-133.

[34] Calic implementation, http://www.ece.mcmaster. ca/ xwu/calicexe/ (accessed: 2017)

[35] L. F. Lucas, N. M. Rodrigues, L. A. da Silva Cruz, S. M. de Faria, "Lossless Compression of Medical Images Using 3-D Predictors", IEEE Transactions on Medical Imaging, Vol. 36, No. 11, 2017, pp. 2250-2260.

[36] ITU-T and ISO/IEC JTC1, High efficiency video coding, ITU-T Recommendation H.265 and ISO/IEC 23008-2, April 2013 (and subsequent editions) 